\title{
Ueber einige Gegenstände der thierischen Chemie ;
}

von Dr. Juliuo Vogel.

Werfen wir einen Blick onf de gromen Fortschritte, welche die nrgnaisehe Chemic in der jüngst vergangenen Zeit gernacht hat, auf die rielen neven Entdeckungen, womit sie In den letzten Jahren bereichert wurde, auf dic Genauigkeit, wamit eine Menge organisther Stoffe ihrer Zusammensetzung mach bestimmi and ihren Eigenschaften nach erforscht worden sind, so mufo es jeden aufmerksamen Beobachter befremden, dalf gerade die 'Thierchemie, derjenige Theil der organischen Vewhindungen, welcher die Hauptrolle im menschlichen und thiorischen Körper spielt, bisher auffallend vernschläfigt w urde.

Des Eiweifs, der Faserstoff, der Farbestofi des Blutes, der Xisestef, der Leim, und die Theile, ans welchen dieser gezogen wird und eine Menge anderer Materien, des thiorischen Körpers sind kaum ihren Hapteigenschaften aach bekannt, nur von wenigen derselben, die chernische Zijsammenseiaung exforecht, fast von keinen die Verbindungen, welche de eingehen, die Verïnderungen, weiche sio dorch den Einfuls anderer Stoffe erleiden, genouer beatimmt. Eine Menge Namen begegnen uns hier, wie Domatom, Schleim, thierisohor Extractivetof a. dgl., mit denen kanm Jemand einen heatinmten Begrifi verbindet, als ebenso viele noekends Koholde und Irrlichter, die den Wanderer out diesem Gebiate nur ire fihren, obne thm den Weg za erhellen.

E. fehlt nicht an eivxolnen trefflichen Arbciten aber einzelne Theile dieses Gebietes, ich erinuere an die umfasende Arbeit der Herren Profoworen Wiebig und W6hler aber die Hamsiare, an dis zahlseichen Untersuchungen von 
Mulder über Eiweifs, Faserstoff, Lein a. \& w., aber sie stehen bis jetzt uoch vereinzelt da, noch fehlt die Verknüpfung det einzelnen Thatoachen anter einander, man vermifst den so wïnsshenswerthen praktischen Einfinfs auf die Kenntnifs der Vorgänge im Organismus.

Und doch ist das genanere Studium dieser Verbindungen fïr diu Phyoiologie wle fïr die praktischo Medizin von der gröfsten Wichtigkeit. Wie ist eine genaue Keuntnifs des Blutes möglich ohne vorhergehende Erforschung der dasselbe konstituirenden Materien, wie eine klare Ansicht von dem Wesen der Verdaung ohne Kenntnifs der Bestandtheile unserer Nahrungsmittel und des chemischen Verhaltens derselben? Wie will man sich lRechenschaft geben von der Veränderung des Urins in Krankheiten ohne eine genaue Kenntnifs ron den Bestandtheilen desselben in normalen Zastande und den Verändertıngen, welche dieselben durch gewisve Einflïsse erleiden?

Freilich stellen sich den Untersuchungen dieser Stoffe eigenthïmliche Schwierigkeiten entgegen. Viele von ihnen krygtallisiren nicht und lassen sich nur sehr schwer rein erhalten; ihre wahre Zusummensetzung, ihr Atomgewicht ist schwieriger auszumitteln, als bei vielen anderen Substanzen, sie sind meist sehr zusanmengesetzt, daher geringe Beobachtungsefehler bei den Analysen derselben viel gröfsere Fiehler bedingen, als anderswo. Diese und ähnliche Gründe waren es whrscheinlich, welche die Chemiker bisher abgehalten haben, sich mehr mit den thierischen Materien zn besclüftigen.

Bei der Schwierigkeit dieser Gegenstände, bei threr Wichtigkeit für Phyoiologie und Medixin mufs aber jeder beitrag zur näheren Kenntnifs derselben erwünscht seyn, wenn or anch nur fragmentariech erscheinen sollte.

In diesem Sinne gebe ich im Folgenden die Reoultate meiner Arbeiten iber einige thierische Stoffe, die ich alle in 
Laboratorium des Hrn. Prof. Llebig anstellte, wobei icb mit Vergnïgen anerkenne, dafs ich mich fast in allen Punkten soines gitigen Rathes zu erfreuen hatte. Sie machen nicht auf Vollständigkeit Anspruch : genöthigt, diese Arbeiten jetzt für läıgere Zeit zu unterbrechen, bin ich nicht im Stande, manche Lücken auszufüllen und mufs vieles noch Wünschenswerthe, Untersuchungen der Verbindungen, Bestimmungen des Atomgewiclits, der Zersetzungsprodukte u. s. w. für spätere Zeiten aufschieben.

Ich werde in Verlaufe der Darstellung öfters Gelegenheit haben, auf Ilerrn Mulder's Arbeiten über dieselben Gegenstände zurïckzukommen; seine Resultate stimmen meist sohr nahe mit den meinigen üterein; wo ich von ihm abweiche, ist es wenigstens nicht ohne gute Gründe und sorgfaltige Priifung geschehen.

\section{Thierisches Eiweifs.}

Mehrmals wurde versucht, die Elementarzusammensetzung des Fiweifses zu bestimmen; es wurde analysirt von Gay-L ussac, Thenard, Michaelis, Prout und neuerlich von Hrn. Mulder, dessen Arbeit bei weitem die genaueste, zuverlässigste ist.

Es schion mir wüuschenswerth, die von ihm gefundene Zusammensetzung durch neue Analysen zu bestätigen.

Ich wählte das Eiweifs von Eiern, weil man es am leichtesten in gröfserer Menge rein erhalten kann, und zwar durch Hitze coagulirtes Fiweifs, weil sich diefs leichter durch Auswaschen mit Wasser von den beigemengten aufïslichen Salzen befreien lärst, als flïssiges.

Das Weifse von hartgekochten Hühnereiern wurde sorgfaltig von dem âufveren Hâutchen und etwa anhängenden Theilen des Dotters gereinigt, in kleine Stuicke zerschnitten und mehrere Tage lang mit Wassar angewaschen, his dieses 
Nichts mehr auszog. Es wurde dann zwischen Fliefspapier bei gewöhnlicher Zimmerwärme getrocknet; nach einigen Tagen war es hart, spröde geworden, blafsgelb, durclischeinend, ja fast durchsichtig, dem Aussehen nach arabischem Gummi vollkommen ähulich, es knirschte zwischen den Zähnen. und weichte in Wasser nur langsam wieder aul. Durch das Trocknen hatte es gegen $90 \mathrm{p}$. an Gewicht verloren. Eis wurde nun zu feinen Pulver zerriebeu und in Wasserbad bej einer Temperatur von $100^{\circ} \mathrm{C}$. noch weiter ausgetrocknet. Diefe wurde natürlich vor jeder Analyse wiederholt, nm das hygroskopische Wasser zn encfornen.

Das so getrocknete und gepulverte Eiweifs wurde mebr mals nit Aether digerirt, um alles Fett darans zu entfernen.

Um die Menge der unorganischen Bestandtheile zu bestimmen, welche mit dem Eiweifs verbunden sind, wurde von dem getrockneten und fein gepulverten Ejweifs im Platintiegel eingeäschert, was sich nur mit grofser Mühe bewerkstelligen läfat.

1,2636 Gr. hinterliefsen 0,0295 Gr. Asche, was genan $2 \frac{1}{8}$ pCt. des trocknen Eiweifses beträgt. Diese Asche bestand gröfstentheils aus phosphorsaurem Kalk und etwas schwefelsaurem Kalk.

Andere fanden eine etwas verschiedene Menge von Asche, Berzeliu 1,8 pCt., Mulder 4,12 pCt.; die Menge der unorganischen Bestandtheile des Eiweifses scheint also nicht immer gleich zn seyn.

Solchen, welche diese Versuche wiederholen, möchte ich rathen, die Eiuäscherung nicht im Platintiegel vorzunehmen, weil die Phosphorgäure durch die gleichzeitig unwesende Kohle beim Glühen leicht zu Phosphor reduzirt wird und dadurch der Tiegel verilorben werden kamı. Man bedient sich daher passender eines Porzellanschälchens, auch ist es vortheilhaft von Zeit za Zeit während des Glühens einige Tro- 
pfen concentrirte Sulpetersânu, oder etwas salpeteruares Ammonisk zazusetzen, um dic sonst sehr schwierige Verbrennung au befördern. Diefs inufs aber sehr vorsichtig geschehen, weil aurserdem durch die eintretende Verpufiung leicht kleine Mengen der Asche herausgeschlendert werden und verloren gehen.

Das auf die erwähnte Weise dargestellte, vom Fett befreite Eiweifs wurde nach Liebig's Methode mit Kupferoxyd rerbrannt.

l. 0,2749 Gr. Eiweifs gaben 0,008 Gr. Kohlengäure and 0,1785 Gr. Wasser.

Da diese Analyse, mit Mulder's Resultaten verglichen, etwas $2 u$ wenig Kohiensäure gab, und dieser angegeben hatte, dafs das Eiweirs mit Kupferoxyd nicht vollständig verbrennt, so wurde eine andere Portion mit chromsaurem Bleioxyd verbrannt. Hierbel gaben:

II. 0,4078 Gr. Eiweifs 0,779 Gr. Kohlensz̃ure und 0,2645 Gr. Wasser.

Der Stickstoffigehalt wurde nach der ron Hrn. Prof. Liebig angegebenen Methode auf indirekte Weise gefunden, indem man das relative Verhältnifo des Stickgases aur entwikKelten Kohlensäure festsetste. $D a$ die erste Analyse eine Abweichurg ron Hrn. Mulder's Hesultaten ergab, so wurde hieranf besondere Mühe verwandt, nm das Resultat 80 genau als möglich 20 erhalten, und recht viele Versuche angestellt.

Bei jedem einzelnen der Versuche wurde vora entwickeltes Gas zu Anfeng und zu Ende eine Probe genommen nnd mit ytmosphz̈rischer Luft gemischt : es zeigte sich niemala anch nux die geringste Spar salpetriger Säure und eo bildete sich also hein Stickstofforyd.

1. D Röhren Gasgemenge gaben im Durchschnitt das Verhailtnifs des Stichgases zur Ruhlensäure gonau wie $1: 7$ II. A Böhren ergaben ein Verhalltuils von 1 : 7,21 . 
Vogel, aber exinige aegenstänite der thierischen Chemie. 2h

III. In 14 Röhren war das mittlere Verhältnifs vie $1: 7,24$.

Da man indefs nicht sicher seyn kann, ob das Eiweifs, welches man auf dicse Weise crhält, wirklich ein chemisch reiuer Körper ist, on salı ich mich nach einer anderen Methode um, es so rein als möglich darzustellen. Die folgende schien das Gewïnschte zu leisten.

Eiweifs von gekochten Hühnereiern mit Wasser wohl ausgewaschen, wurde in verdünnter Kalilauge aufgelöst. Was löste sich leicht. Die filtrirte Lösung wurde mit Schwefelsāure übersättigt, und dadurch das Eiweifs als ein zartes Magma vou rein weifser Farbe niedergeschlagen. Die Masse wurde auf ein Filtrum gebracht und mit destillirtem Wasser so lange ausgewaschen, bis das ablaufende Wasser nicht mehr sauer reagirte und, auf dem Platinbleche verdampft, nach dem Glühen keinen Rückstand hinterliefs. Diefs Auswaschen erfordert ziemlich lange Zeit: man erleichtert sich die Arbeit, wenn man die Masse manchmal mit destillirtem Wasser anrülırt und damit einige Zeit digerirt.

Das ausgewaschene Eiweifs wurde im Wasserbade bei $100^{\circ}$ getroch net.

Das so dargestelite Eiweifs enthält keine Schwefelsäure mehr; beim Verbrenuen mit kupferoxyd entwickek sich keine Spur schweniger Säure, von der sich doch schon die geringste Menge durch den Geruch wahrnehmen läfst.

Ws lieferte anf die oben angegebene Weise verbrannt, Stickstoff und Kohlensäure im Verhältnifs von $1:$ z,82, drei Röhren unter $\boldsymbol{\gamma}$ gaben genan diesee Verhältnifs.

Um die Menge des Kohlenstofle und Wasserstoffe 24 bestimmen, wurde es mit Kupferoxya verbrannt; Hr. Gnindelach hatte dio Güte diefs zu thun.

1. 0,439 Gr. Eiweifs gaben 0,8042 Gr. Kohlenakure and 0,2887 Gr. Wasser. 
Diefs ist etwas zn wenig Kohlensäure, wovon wir uns den Grund wohl erklären können. Am Ende der Analyse nämlich hatte sich der hintere Theil der Verbrennungsröhre verstopft, so dafs die noch in der Röhre befindliche geringe Menge von Kohlensäure nicht anf die gewöhnliche Weise durch Einziehen von Luft in den Kaliapparat getrieben werden konnte, und also verloren ging.

11. Bei einer zweiten Analyse mit Kupferoxyd gaben 0,4479 Gr. Eiweifs, 0,840 Gr. Kohlensäure und 0,273 Gr. Wasser.

Berechnen wir nun aus diesen verschiedenen Analysen die Menge der einzeluen Elementafbestand theile des Eiweifses nach Procenten, so erhalten wir Folgendes ;

Eiweifs iu Kali gelöst and durch Schwefels. gefïllt.

I.

II.

I. $b$.

II. b.

Kohlenstoff 51,228 - 52,817 - 50,654 - 51,856

Wusserstoff 7,232 - 7,206 - 7,055 - 6,766

Stickstoff *) 16,465 - 16,970 - 16,022 - 16,403

Snuerstoff 22,745 - 20,678 - 23,939? - 22,645?

Asche $\frac{2,330-2,330-2,330 ?-2,330 \text { ? }}{100,000-100,000-100,000}$

Bei dem durch Kali und Schwefeleäure dargestellten Eiweifs kounte ich ans Mangel an Substanz nicht bestimmen, wieviel es Asche hinterläfst; es bleibt aber nach dem Verbrennen eine geringe Menge einer weifsen Asche zuriick.

Aus den angefiihrten Mnalysen geht aber hervor, dafs diefs mach beiden Methoden dargestellte Hiweifs dieselbe chemische Zusammensetzung hat: die Resultate der Analysen I und II b. stimmen wenigstens sehr nahe ïberein.

Ebenso kommen diese Resultate, den Stickstofigehait aus-

*) Das Verhältuirs von $1: 7,21$ angeuoinmen. 
genommen, sehr nahe mit deu Ergebnissen von Ifrn. Mulder's Analysen überein. *)

Mulder hat aus seinen Analysen eine Formel für dae Eiveifs berechnet, sie heifst:

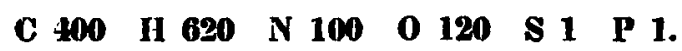

Es möehte diefs aber noch zu früh seyn; ich halte es fïr besser, damit noch zu warten , bis wir das wahre Atomgewicht und die Zersetzungsprodukte dieses Körpers genauer kennen.

Ich habe auch, wie Mulder, ein Kupfersalz des Eiweifses dargestellt. Es wurde auf folgende Weise erhalten:

Flüsaiges Eiweifs von Hühnereiern wurde mit destillirtem Wasser vermischt und filtrirt. Das Filtrat mit einer $\Delta$ uflösung von schwefelsaurem Kupferoxyd versetzt gab einen hellblaugrünen Niederschlag, der auf ein Filtrum geworfen und so lange mit destillirtem Wasser ausgewaschen wurde, bis die ablaufende Flissigkeit durch Ammoniak nicht mehr geblïut wurde. Man verliert zwar durch dieses lange Auswaschen etwas von dem Niederschlag, weil er nicht ganz in Wasser uuauflöslich ist, erlält ihn aber $\mathrm{nm}$ so reiner. Er wurde bei $100^{\circ} \mathrm{C}$. im Wasserbade getrocknet und fein gopulvert.

Dieses Produkt iot nicht, wie Mitscherlich angibt, eine Verbindung von basisch schwefelsaurem Kupferoxyd mit Elweifs, Bondern, wie schon Mulder fand, ein Eiweifssals des Kupferoxyds, ein Kupferalbuminat. Fis enthält, wohl ansgewrachen, keine Schwefelsäure; denn, wenn es in Sadpeter-

-) Mulder giebt die Zusammensetrang des Eiweifses an: C 64,48. H 7,01. N 15,70, 0 22,00, \& a. P 0,81. Das ununittelbare Resultat sainer Analye int sber C 52,43 H 6,73 N 18,30 O 21,43 Asche 4,12. Die obige Formel lst dadurch eutstauden, daf er statt 4,12 pCt. uuorganischer Bestaudtheile nur 0,81 pCt.; als dle im Eiweils euthalteue Menge von Bchwefel and Phoophor fit constant annahm. 
sänre gekocht wird, entsteht durch salpeterennren Baryt kaun eine Spur eines Niederschlags nnd beim Verbrennen mit Kapferoxyd entwickelt sich keine schweflige Säure.

U'm zu wissen, ob diese Substanz das Giweifs in unverändertem Zustande cuthält, wurde untersucht, in welchem Verhältnifs der Kohlenstoff und Stickstoff in dieser Verbindung enthalten ist. Sie wurde mit Kupferoxyd mach der öfter erwähnten Methode verbrannt.

1. D Röhren ergaben das durchschnittliche Verhältnifo des Stickstoffs zur Kohlensäure wie $1: 7$.

II. 14 Röhren ergahen ein Verliältnifs wie $1: 7,333$.

Es geht also daraus hervor, dafo das Eiweifs unverïndert in diese Verbindung nit Kupferoxyd eingeht.

Die Verbindungen und Zerset znngsprnitukte des Eiweifses sind sehr mannigfaltig, aber hisher mit wenigen Ausnahmen uur sehr oberflïhlich, mejst blos den Iteartionen nach bekaunt.

Siuren scheisten je nach ihrer Concentration und nach der dabei angewanlien 'lemperatur mit dem Eiweifs sehr verschiedene Produkte zu bilden. So z. ib. die Salzsäure.

Wird viel liwweifs mit concentrirter Salzsäure gekocht, so erlült man eine sclimutzig weifse Masse; die ïberstchende Flüssigkeit ist braun, trübe und enthält organische Materie aufgelöst.

Wenig Eiweifs mit einem Ueberschufs ron concentrirter Salzsäure gekochıt, Iöst sich vollsiändig. Dic Auflösung nimmt bein: Erhitzen allmählig eine schöne Lilafarbe an: durch $\mathbf{Z a -}$ satz von $W$ asser erhält man einen weifsen Nivderschlag.

Wird Eiweifs mit einer Mischung von gleichen Theilen Salzsäure und Wasser bei einer Temperatur von $70-80^{\circ}$ längere Zeit digerirt, so erhält man eine dunkelbraune aber klare Flüssigkeit, unanfgelöste Stiiche Eiweifs haben ebenfalls eine dunkelbrane Farbe und eine gailertartige Beschaffenheit angenommeu. 
Lafat man eine noch mehr verdünnte Säure anf Eiweifo wirken, so erreicht man eiut Mischung, wo letzteres mit unveränderter Farbe und vielleicht uuch mit unveränderter Zusammensetzung aufgelöst wird. Diese Auflösung hat grofse Aehnlichkeit mit dex Auflösung des Eiweifses durch küngtliche Chymification, tritt aber bei einer 'Temperatur ron $60-70^{\circ}$ erst nach mehreren Tagen ein.

Eine sehr verdïnnte Säure, 10 - 15\%'ropfen Säure auf 1 Unze Wasser, löst Eiweifs auch bei wochenlanger Einwirkung nicht auf. Sie zieht nur Kulksalze aus dem letzteren au, ohne dieses selbst auf wahruehunhare Weise zu verändern.

Man sieht aber aus diesen kurzen Angaben, dafo dieser Gegenstand selur rerwickelt ist und nur durch fortgesetzte mühsame Untersuchungen aufgehellt werden kann.

Durch verdünnte Kalilauge wird das Fiweifs, wie schon erwähnt, uırerändert aufgelöst, und durch Säuren daraus niedergeschlegen.

\section{Fasersloff.}

Ich bediente mich zu meinen Untersuchungen des Faserotoffs aus Ochsenblut. Das Plut warde geschlagen, der in feinen Fasern und Fäden geronnene Faserstoff so lange mit Wasser ausgewaschen, bis dieses ungefärbt ablief. Der so gereinigte Faserstoff hat eine bläulichweifse Farbe, wie abgerahnte Milch. Er wurde getrocknet, erst bei gewöhnlicher Zinmerwärme zwischen Fliefspapier, dann bei $100^{\circ}$ im Waserbade, fein gepulvert, mehrmals mit Aether ausgezogen, dann wieder getrocknet.

Um dis Menge der Asche zu bestimmen, welche ex nach dem Verbrennen hilıterläfst, warde er im Porzellanachailchen rerbrannt, und dabei, um das Einäscherı zu befördem, vorvichtig concentrirte Salpeteroäure zugesetat. 
spribglich enthaltene Wasser eine whitigo Holle in seinor Constitution spielt und daf das basioche Sala angensebcindioh zersetzt wird, wenn es in waserf freies Salz übergeht.

Die Aloune bilden die wichtigste Kiuse der schwefeisatren Salze; ich hatte aber nie davon an meiner Disposition, am Thre Constitution mit der der seliwefelsauren Ataunerde zu vergleichen, die nur schwierig kryetallisirt erhallen werden kann. Das im Kalialann enthalifne Wniser rermindert sich durch Erthorescenz in einem Danpfbade bei $65^{\circ}$ auf 6 Atome; seine Formel ist hierwoch $\mathrm{kO}, \mathrm{SO}_{3}+\left(\mathrm{Al}_{2} \mathrm{O}_{3}, 3 \mathrm{sO}_{3}+6 \mathrm{sq}\right.$. +18 aq.) Wine Analyse des Natraualanus ergab mir, dars dieser, wie der Kalizlaun, 24 Alome Wasser, und nicht $\mathbf{2 8}$, enthalte.*)

\section{r. Von den Chlorïrest.}

Die Verwandtschaft der Wassemstolfsïuren zum Wasser ist nicht bedeutend; wir kennen keine Hydiote der Solzwinte, da die Flüchicheit der letateren die Bildung und Intersuchung ookher Hydrate rechindert; es ist aber wahrseheinlich, dafs sie den Hydratew des Cilmrinagnesiuns etc. enisprechen. Die Chlorẗre ans der Klasse lec Magnesianxyde halten imancr und ziemlich siark 2 At. Wasser enrïck, die nit als salinisches Wasser betrachten künnç. Su krystallisirt das Kupfenchlorid mit 2 Atomen Wusser und niclit mit weniger; mehrere Chlorime dieser Klasse enthalien aber 2 oder $\$$ Atome Wasser mehr, whei die Proportion des Wassers imuer ein Multipel von awei ist.

1. Kupferchlorid; $\mathrm{Cu} \mathrm{Cl}, 2$ aq. Die blanen prisulischen Krystalle dieses Sulues werden bei einer Temperatur, die den Siedpnnkt nicht übersthreitet, braun und verlieren den gröfsten Theil ibre Wassers. Is Grain verlaren in si-

- Aunal. d. Pbarm. Bd. XXIIL. 8. 268. 
nef viel hïheren Temperalur 3,22 Oria Wasser und binterliefoen 11,7t Kupferchlorid, welches der Luft sugexelat beld wieder 3,16 Groin Hasace auluabua elesuse die urspriingliche blave Purbe wieder eilielt. Ich glumbe, dab durch diew Art der Wiederaulinalume des Wamers oft lfydrate eutotehen. de. ren Zusammenstzung defwregen genauer iat, als die aus Auflösungen dargesteflien, weil hier kein mechaniech in den liryallen eingewhlossence Waser vorthanden ist, wie dies oft vorkonimil.

2. Mnnganchlorür; $\mathrm{MnCl}, 8$ aq. +2 aq. 15,53 Graias der feivebfiarbenew Kirystaile lieferten 29,57 Chlorsilber, entoprechend a,s Maugauchlorü. Der Verfust, 5,01 Groin, driekl den Wracergehalt, der 26,12 Prec. betrigt, aus. Beim 'Trwokeen bei $100^{\circ}$ an der Luft, oder in Vacuo über Sclurefelalure bei gewöluulicher 'iemperatur, verliert das Salz die IBIthe seines Nascerm.

8. Livenchlor är; le $\mathrm{Cl}_{2}, 2$ थ. +2 oq. Nuch drei mil diecen Salze augestellien Aualywen betrïgt de- Kakergohalt

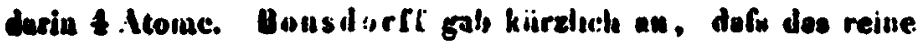
bala, aus eimer sanren Aullowang k rystallinirt, of Alonie Wyaser outhalte.

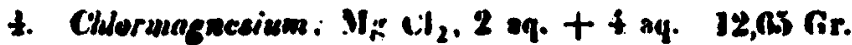
Lryotallicirtes Salz elithallen 1,20 (:blor $-23,01$ p. c.; diefe

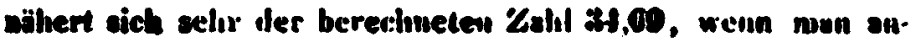
wiment, dafs es 6 At. Wewer entliäls.

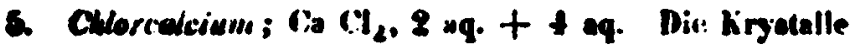
diewes serniefslichen Salzes enlinalcen, nuch dem Irocknen in Vecuo, bie sur antungenden bifllorestcuz, fi. Wasser, wir. man diefs gewöhulich anuimont; werden sie abe: wälurend der Beifoen Jahreascit 10 'lage lang im teereu Ranm ïber Schwefelsiute gelasen, so werden sie undurchsiontig und gläncend vie Tult, ohne su zerfallen; dex Waswergehait betrïgr ajodann nor woch 2 Atome. 


\section{Kïscstof.}

Ich wählte zur Darstellung des Käsestoffs die ron Berselius angegebene Methode, wobei man denselben durch verdünnte Säuren aus der Milch abscheidet, do diese ihn am reinsten zu liefern verspruch.

Da das in grofser Menge in der Milch enthaltene Fett das Auswaschen und Reinigen des Käsestofis sehr erschwert, wie ich bei melireren Versuchen fand, so bediente ich mich der Buttermilch, aus welcher der gröfste Theil der Butter besser und rollständiger, als es durch blofses Abralumen geschehen kann, entfernt ist.

Die Buttermilch wurde mit verdïınter Schwefelsäure versetat, mit der sich der Käsestoff verband und beim Er. wârmen als weifses. käsiges Magma niederfiel; die Molken wurden abgeseihi, der scliwefelsaure Käsestof :nit destillirtem Wusser zerrïhrt, damit digerirt und aut eineu Filtrun ausgewaschon. Diefs Verlahren wurde 80 oft wiederholt, bis das ablaufende Waschwasser auf dem Platinblech verdampft keiuen Rückstand mehr hinterliefs.

Nach dem Auswaschen wurde der schwefelsaure Räsestoff in destillirtem Wasser zertheilt, mit kohlenszuren Baryt, angerïhrt und digerirt, wohei sich die Schwefelö̈ure mit dem Baryt verbindet nud der Käsestoff sich im Wasser löst. Es wurde fitrirt und dic wässrige Lösnng im Wasserbade bis zur Trochne abgedampft. Das $\mathbf{\Lambda b g e d a m p t t e ~ s t e l l t c ~ e i n e ~ w e i f s e ~}$ oder schwach gelbliche, durchsichtige Masse dar, wie arabisthes Gummi, dem sie auch darin glich, dafs sie mit wenig Wasser eine schleimige Flüssigkeit bildete. Die trockne Masse wurde gepulvert, mit Aether digerirt, um anch die letaten Spuren ron Fett auszaziehen, dann bei $100^{\circ}$ getrocknet.

Um die Menge ler in diesem gereinigten und getrockneten Käsestoff eathaltenen unorganischen Bestandtheile an 
beetimmen, wurde etvas daron in cinem Porzellanschälchen verbrant, und die vollständige Sinäscherung durch tropfen. weisen Zusatz von concentrirter Salpetersäure befïrdert.

0,6805 Käsestoff hinturliefsen nack: dem Verbrennen $9,1,4$ Gr. Ascht, welche au ciner weifsen Masse mit einem schwachen Stich ins Grünliche schmolz. Diefs gibt die enorme Menge ron 21,454 pCt. Aache.

Mit Kupferoxyd rerbranut gab der Käsestoff folgendo Reanltete :

1. 0,4836 Gr. Substana gaben 0,24n5 Gr. Wasser. Die Bestimmung der Kohlensäure bei diesen Versuche wurde vereitelt, da am Ende der Analyse etwas Kalilange verloren ging.

II. 0,3552 Gr. Sabstanz gaben 0,5230 Kohlenstiure und 0,1844 Wasser.

11!. 0,3802 Käsestoff lieferten 0,367 Gr. Kohlensäure nad 0,210 Gr. Wasser.

Der Stickstoffgehalt wurde auf die mehrerwähnte inclirekte Weise bestimunt:

I. Böhren gahen als Mittel ein Verhïltnifs vou $\mathbf{S}_{4}^{4} \mathrm{i}$ ? stoff zu Kohlensäure wie $1: 7,49$.

II. 15 Höhreu gaben ein Verhêltnifs wie $:: 7,51$.

Das wahrochcinliche Verkältnifs ist also yie $1: \frac{7}{2}$.

Daraus ergiebt sich folgende procentische Zusammetssetzung :

1. II. III.

\begin{tabular}{|c|c|c|c|c|c|}
\hline Rohlenstolf & - & - & 40,713 & - & 41,234 \\
\hline Wasserstoff & 5,733 & - & 5,788 & - & 6,135 \\
\hline Stickstoff & - & 一 & 12,570 & - & 12,720 \\
\hline Sauerstoff & - & - & 19,485 & 一 & 18,457 \\
\hline \multirow[t]{2}{*}{ Asche } & - & - & 21,454 & - & $21, \pm 54$ \\
\hline & & & DOO & & $0 x$ \\
\hline
\end{tabular}




\section{$34 V_{0} e l$, uber einige Gogenstände der thieriuchon Chomis.}

Von der objgen Asche löste sich Nichts in Wassef, sie enthielt also keine alkalischen Salze; dagegen löste sie sich vollständig in Salzsäure unter Aufbrausen.

Aus dleser Auflösung wurde durch Uebersältignng mit Amnoniak phosphorsaurer Kalk in bedeutender Meuge gefällt: Die voin Niederschlag abgegossene Flïssigkeit gab mil doppelt oxalsaurem Kali noch einen Niederschlag.

Durch Schwefelsäure entstand in der sauren lösung ein Niedersching, der sich in vielem Wasser nur zum Thril löste, und Gypslösung bewirkte gleichfalls einen Niederschlog. Die Asche enthielt also aufser phosphorsaurem und kohlensaurem Kuik auch kohlensauren Baryt, der jedenfalls bei der Bereitung des Käsestoffs erst hinzugekommen war, wiewohl die mit kohlensaurem Baryt digerirte F'liissigheit zweimal filtrirt wurde unt immer ziemlich hell durch dus Fittrum liel.

Defshalh kann die obige Aschenmenge nicht als die normale betrachtet werden. wiewohl diese Verunreinigung durch Baryt in dem Resultat der Analyse nur auf den Kohlenstoffgehalt einen geringen Ëinfluls ansïben hann.

Betrachten wir aber die Zusammensetaung des Käsestoffy, ohne auf die Asche Riicksicht zu nehmen, so erhalten wir:

I. II. III.

$\begin{array}{llll}\text { Kohlenstoff } & - & -51,86 & -59,58 \\ \text { Wasserstoff } & 7,29 & -7,36-7,82 \\ \text { Stickstoff } & - & -26,01-16.21 \\ \text { Sauerstoff } & - & -24,77-23,45 \\ & & 110,00-100,01 .\end{array}$

Nach diesen Angaben des Thatbestaudes erlaube ich mir einige kurze Bemerkungen über das Verhältnifs, in welchem diese drei Körper zll einander stelien. 
Alle drel Substanzen, Eiweifs, Faserstof und Käsestof, haben in ihren Eigenschaften sehr vicles Gemeinsane; sio sind alle eines doppelten Zustandes fihig, des aufgelösten und des geronnenen. Hierbei findet nur der Unterschied statt, dafs der Faserstoff ron selbst gerinut, sobald er aus dem Körper kommt, der Käseatofi in seinen geronnenen Zustand durch das sogenannte Larb anf eine bisher noch unbekannte Weise rersetzt wird und die Geriuung des Eiweifses vorzüglich durch Hitze hervorgebracht wird.

Alle drei theilen ferner die Eigenschaft, in geronuenen Znstande bein Kochen mit einem Ueberzchufs von concentrirter Salzsäure mit schöner Lilafarbe aufgelöst zu werden; eine Eigenschaft, die sehr wohl benützt werden kann, um sich durch einen einfachen Versuch von der Gegenwart einer dieser Stoffe im geronnenen Zustande zu übsrzeugen.

Betrachten wir nbor die Klementarzusammensetzung dieser 3 Körper, abgesehen von den unorganischen Bestandtheilen, welche beim Verbrenucu als Asche zurückbleiben, so finden wir, dafs sie fast genau dieselbe ist. Sie ist die folgende :

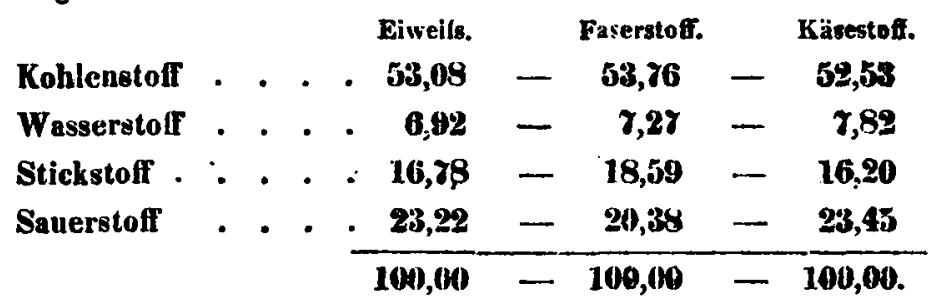

Diese Uebereinstimmung erscheint noch gröfster, wenn man wicht, wie es hier geschehen ist, die Resultate einzeluer Analysen, sondern die Mittelzahlen aus den Resultaten meinrerer ats Basis der Vergleichung annimmt.

- Ich glaube mich aber durch die angeführten zahireichen Analysen zu dern wichtigen Schlusse berechigt, den Mulder bereity fïr Eiweifs and Faserstoff gezugen, dafs Kiweifs, 
Paserstoff und Käsestoff im Thierreiche in derselben innigen Vervandlschaft mit einunder stehen, wis Zucker, Stärko und Cummi in Pfanxonreiche, eine Beobachtung, welche, weiter verfolgt, die wichtigsten und interesseutesten Aufschlïssc über so viele Vorgänge im thierischen Organismus zu geben verspricht.

Merkwürdig ist feruer, dafs das Verhältuifs des Sticksioffis zum Kohlenwtoff bei diesen 3 Körpern in einer Art regelmülsiger Progression zu steigen scheint. Der Faserstoff enthult den meistcn stichsti)ff. Ilier ist das lerfïltuifs mie 2: $6 \frac{3}{7}$; beim Eiweils, welches zunächst folgt, wie $2: 7 \frac{1}{3}$. Beim häsestoff ist der Stichstofigehalt am geringsten und das Verhältuil's wie $2: \boldsymbol{\gamma}:$.

lch wage es uicht, aus der prozentischen Zusammensetzung dieser hörper eine formel zu berechuen, wie Mulder es gethan. Es kommen hiebei eine Mrnge Punkte in Butracht, die vorher gelöst werden müssen: hieher gchört die Frage, welche der worganiachen Bestandtheile wesentich aur $\mathbf{Z u -}$ sammensetzung dieser Körper gelüren. Mulder nahn an, dafs nur Phosphor und schwefel dem Eiveils und Fascrstoff wesentlich sey: aber kann man nicht init demselben Rechte anch die Kalksalze als wesentlich zur 'Cusamuensezzung gehörig reklamiren? Teberdiefs könnten wir bei einer so hohen Formel, wie dic von Mulder berechnete ist, aúch bei den beste: Analysen nie sicher sey", ob wir uns beim Wasserstoff z. B. uicht un rolle $30, \mathrm{ja}$ to . Atoue irren?

Nur forfgesetate Untersurhungeu über das Alomgewicht dieser Substanzen, ihre Verbiudungen und namentlich ihre \%ersetzungsprodukte kömuen uns hierin zu einer bestimmten Erhenntnifs führen, und es wäre zu wünsclien, dafs sich ein Chemiker speziellcr damit beschältigte. Freilich wären jahrelange, mülısine Bntersuchungen nothwendig, un eine ebenso vollendete Arbcit zu liefern, wie die der Herren Prof. Li ebig 
und Wöhler über die Ilarnsäure, uber der Gegenstand ist es wahrlich werth: er hat gewils oine gröfsere Wichtigkeit für die Kenntnils des thierischen Körpers nnd die in inm vorgehenden Prozesse, rerspricht gröfsere Auf́chlüsse für Fhysiologie and Medizin, als irgend ein anderer Gegenstand im ganzen Gebiete der Chemie.

\section{Künstliche Chymification.}

Eberle hat vor einigen Jahren die intercssante Entdekkung gemacht, dals die Flüssigkeit, welche man durch Digestion des Luabmagens rom Kulh oder Rind mit sehr verdïnnter Salzsäuce erhält, die Eigenschaft hesitzi, die meisten Nahrungsmittel ebenso aufzulösen, wie der natürliche Magensaft, und sic in cilte dem Chymus ganz ähnliche Masse zu verwanticlis. Vergleichende Versuche an Thieren zeigten, dafs dic Speisen dureh die kïnstliche Chymification ganz dieselbe Veründerung erleiden, wie durch die natürliche in Magen; dic Versuche ron Beaumont in Amerika haben diefs auch für den Menschen nachgewiesen.

Durch späterc Intersuchungen Anderer, vorzüglich durch die ron Joh. Nliller und Schwann wurde die obige Entdecknne bestätigi nnd als cine für die Physiologie der Verdaunng wichtige 'Thatsache auerkannt.

Ich habe eine Reihe von Untersuchungen über diesen Gegenstand angestellt, die aber gröfstentheils mein Interease fir den Physiologen, ais für den Chemiker haben, von denen ich dàher hier nur die ausführlicher mitheilen will, welche sich auf die Umãnderungen beziehen. die mehrere organische Materien, vorzüglich Fiweily und Faserstoft, durch die künstliche Cliymifikation erleiden.

Doch mufs ich den Vorgang bei ner hünstlichen Chymif kation und die danu nothwendigen Bedingungen, wie sie nuF 
34 Vngel, ïber cinige Gagenstande der thierischen Chomie.

den früheren Untersuchungen und meinen eigenen het vorgehen, wcrigstens kurz roransschicken.

Durch Ilitze geronnenes biweifs erleidet, wie schon oben erwähnt wurde, durch Säuren rerschiedene Veräıderungen, welche verschiedell sinıl nach der Natur der Säure, ihrer Menge und dem Grade ihrer Verdiinnung. Eine sehr verdünnte Salzsäure, die nur norh saner schmeskt, haum die Zähne stuıpf marlit, löst dasselbe bei gewölnnlicher Temperatur auch bei woshenlanger Hinwirkung nicht auf. Bei einer etwas höheren Tenperatur dagegen, bei ro $-80^{\circ} \mathrm{C}$. löst sic Riweifs, und zwar ohne Verānderung der Farbe; doch siud dazn immer mehrere Tage erforderlich. Künstliche Verdaunngsflissigkeit dagegen, welche Salasäure in derselben Verdïnuming enthält, löst Eiweifs bei einer Temperatur von 30 - $40^{\circ}$ schon uach einigen Scunden ant; ebenso Faserstoff, rohes und gekochtes Fleisch, kurz die meisten Speisen.

Man bereitet sich die künstliche Verdauungeflüssigkeit am besten und reinsten, indem man den vierten Magen eines Kalbes oder Kindes, den sngenannten Laabmagen, mit reinem Yasser abspült, die Schleimhaut abpräparirt, mit dem $4-5$ fechen ihres fewichtes Wasser ihbergiefst und so viel Salzsïure zusetzt, daîs die Flïssigkeit deutlich saner sclınierkt. Man digerirt das Ganze 8-12 Stunden lang bei einer Temperatur ron $30-40^{\circ}$, (Kochhitze vernichtet die verdauende Kraft) und filtrirt. Die abfiltrirte Flüssigkeit zeigt die verdaneide Kraft, ja sie zcigt diese auch noch ebenso stark, wenn sie mit der 3 - 4fachen Menge schwach gesäuerten Wassers verdiunt wird.

Dje Natur deṣ bei der künstlichen Chymifikation wirksamen 'rinzips, die Art seiner Wirkung ist durchaus unisekannt. ibsan hat den Vorgang mit der Gährulig verglichen, aber diefs neifst cin Räthsel durch ein audercs löseu, man hat einen eigenthü:mlichen Ssoif angenommen, der diese Wirkung haben 
Vogel, über einige Gegenstände der. thierischen Chemie. 39

sollte, hat diesem sogar den Namen Peprin gegeben, aber er ist durchaus hypothetisch, und ist noch niemals isolirt dargestellt, vielweniger analysirt und seineii chemischen bigenschaften nach bestimmt worden. Ich will indessen die lixistenz einer cigenthümlichen, bei der Chymifikation wirksamen Materie damit nicht in Abrede stellen, nur kennen wir sie noch nicht. Man kann zwar durch einige Reagentien, z. B. durch neutrales essigsaures Blei, durch Puecksilberchlorid Stoffe aus der Verdauungaflïssigkeit nicderschlagen, wejche nach der Abscheidung des Metalles durch Schwefelwasserstofi die verdanende Kraft noch zeigen, aber die zusammengesetzten thierischen 'Theile, wie Muskel, Schleimlıäute, enthalten eine solche Menge verschiedener Materien, welche durch cin und dasselbe Reagens ans ihren Auflösungen gefält werden, dafs es gegenwärtig schr schwer, ja unmöglich seyu wiirde, das Pepsin zu isoliren.

Jedenfalls ist dieser Stoff sehon im Minimum wirksam; denn eine Verdanungsflüssigkeit, weiche weniger als ein $\mathrm{pCt}$. feste Kestandtheile enthält, verdaut eine ziemlichı Mengo Eiweifs.

Das verdauende Prinzip bildet sich nicht etwa durch die Hinwirkung der Săure auf die Schleimbaut des Magens, es wird schon durch blolses Wasser ausgezogell. Wird nämlich dic Schlcimhaut mit hlofsem Wasser digerirt, und die Säure erst dem Filtrat zugesetzt. so verdaut dieses gleichfulls.

Auch andere Säuren, nicht blos Chlorwasserstoffsüure, haben den erwähnten Erfolg. Ich versuchte es mit Schurefelsäure, Essigsäuro!, Phosphorzäure und Salpetersäure; alle verdanten, die Phosphorsänre am besten, die Salpetersäure am wenigsten; ein Theil der letzteren rerband sich mit dem Hiwcils und fäbte es gelb.

Nach diesen allgemeinen Bemerkungen komme ich zu den Veriaderungeu, welche geronnenes Biweifs und Faserstoff 
durcîn die künstliche Chynifikation erlefden. Eberle hatte gefuncien and Sch wann es begtätigt, dafts das in der Verdau-

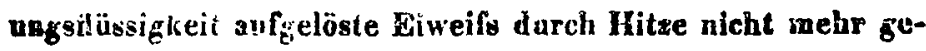
rinne, dafs es zum 'lìeil in Weingeist löslich sey. Beide nehmen an, ts werde in Osmazom und Speichelstoff ungewenteît. Wird nämlich das Filirat, welches das verdante Eiweî́s enthäıt, cur Trovkne abgedampft, so ist ein Theil davon in Weingeist lïolich; die durch den Weingeist anzziehbrre Substanz iat auch in Wasser löslich und wird aus dieser wãsstrigen Lösung durch Gerbestoff und salpetersaures Silber Eefallt; diefs zollte Osmazom zeyan. Wer in Weingeist unlösliche Theil löst sich̆ in kalteñ Wasser, dic Auilösung wird dareh assizssures Biei, Gerbestoff und salzsaures Zinn nur schwach getrïbt; sie hielten diese substanz für Speichelstoff.

Aiver Ostazzum ist ein so ubbestimmter Begriff, dals eigeatichi Nismand xelit wciis, was man sich darunter denken soll, eine tezoichnung, die alus der Clemic liebcr ganz yerbanut widdcit snllic; Past ebensu verhält es sich mit dem Speicheistofi, eis Nanne, womit verschiedene Chemiker ganz von einanior verschiedene Substanzen bezeichnen.

Ueberdiefs mufs es sonderbar erscheinen, dafs das genossene Liweif́s in Osmazom und Speichelstoff ungewandelt wer. den sull, wenn man erwägt, dalis es kurze Zeit hernach in den Chyiusgefursen des Darmkanals vieder als walires Eiweifs angatroffen wird.

Daker schien mir die obige Deutung and Benensung der neugeilaceten Materien Fehlerhaft, so sehr icli von der Richtigkeit iner Weohachtungen jerer beiden verdienstvolien Forscher iberseugt war, wnd ich suchte durch fortgesctate Hutersuchuagen 2 a einer genaneren Kenntnits deo Gegensiandes zu gelangell. Sis gaben foigende Resuitate:

Die iait Chlorwasserstofiäure bercitete, also gaure Verdurusgafiuaigkeit hinterlitist aut dem Platinblech abgedempft, 
eine ziemliche Menge eines Rürtkstandes, der sich beim weiteren Erhitzen schwärat und beim Verbrennen den eigenthümlichen Gestank stickstofthaltiger thierischer Materien entwickelt. Nach dem Glühen bleibt eine geringe Menge einer weirsen Asche zuriick.

Die filtrirte Flïssigkeit erleidet durch Kochen keine Verändernug.

Bei der Neutralisation durch kohlensaures Natron entsteht weder Niederschlag noch Trübung.

Durch Kaliumeisencyenür, Gerbestoff und Alaunlösung wird Nichts gefällt.

Durch schwefelsaures Kupfernxyd kaum eine Spur von Trübung.

Durch neutrales essigsaures Bleioxyd und saipetersanres Siberoxyd weifse Niederschläge, die anfser Chlorblei und Chlorsilber auch noch organiselie Materien enthalten.

Wird gekochtes Eiweifs init V'erdauungstlissigkeit bei einer Temperatur von $30-40^{\circ}$ digerirt, so ist es nach $4-5$ Stunden an den Rändern durchscheinend, und an der gunzen Oberfläche schmierig und breiartig geworden; nach $8-12$ Stunden ist es gewölnnlich zu einer trïblichen Flüssigkeit aufgelöst, welche auch durch wiederholtes Fijtriren nicht klar erhalten werden kann. Unter desn Miik roskope sieht man, dafs diese Trübuug von sehr kleinen farblosen Köruchen herrührt (die meisten haben weniger als $\overline{1}^{\circ} \dot{0}_{\overline{0}} \overline{0}^{\prime \prime \prime}$ im Durchmesser), weiche theils einzeln, theils zn gröfseren Mazsen aneinander gereiht, in der Fliissigkeit schwimıen.

Die Flüssigkeit reagirt natïrlich sauer. Darch Kochen wird sie uicht verändert.

Durch Weingeist enisteht eine geringe Trübung.

Durch Gerbestoff ein reichlicher schmutzig weilsirauner Niederschlag.

Darch Kaliumeisencyanür ein voluminöser weifser Nieder- 
schlag. Durch Kaliumeisencyanid ein reichlicher hellgrüner Niederschlag.

Durch Neutralisation mit kohlensaurem Natron ein galJertartiger weifser Niederschlag, der eich aber durch Filtri: ren nicht trennen läfst, indem er gröfstentheils durch das Filtrum geht, oder dasselbe verstopft, und beim Schiitleln der Flüssigkeit sich wieder in derselben zertheilt. Kir ist in Wasser und gröfstentheils, doch scliwieriger, auch in Weingeist liislich.

A wh nach der Nentralisation durch Alkalien wird die Flüssigkeit moch durch Gerbestofl gefällt, aber nicht inehr durch Kaliumeisencyanür und-cyanid.

Sie wird ferner gefäilt durch neutrales und basisch-essigsanres Bleioxyd, so wic durch Alaunlösung. Durch Quecksilberchlorid entsteht ein reichlicher weifser Niederschlag.

Durch schwefelsaures Kupferoxyd ein reichlicher blaugrüner Niederschlag.

Da mehrere dieser Metallsalze in der ursprïuglichen Verdauungsflïssigkeit keinen Niedersching bewirken, so mutsten die in diesem Falle gebildeten Niederschlïge Verbindungen der Metallsalze mit dem durch dic Chymitication reränderten Fiweifs seyn. Die Untersuchung eines dieser Niederschläge schien daher der beste Weg, um übry die Natur der vorgegangenen Veränderung Aufschlufs zn erhalten.

lch wähte dazu den Niederschlag durch schwefelsaures Kupfer.

Ex hat cinige Scluwierigkeit, diesen Niederschlag rein und in hinreichender Menge zu erlatten. Man mufs die Flüssigke: durch tropfenweisen Zusatz einer sehr verdiunnten Kalilauge genau neutralisiren, ehe man das sehwefelsture hupferoxyd hinzufiggt ; ist sie sauer, so crhält man keiuen Niederachleg, oder man verliert ihu bein Filtriren und Awowa-echeu fast gäuzlich, weil er sich in freier Sïare auflöst; - 
$\nu_{\circ g}$ l, aber einige Gegenstände der thierischen Chemie. 43

int sie alkalisch, so wird der gröfte Theil des Knpfers als Kupferoxydhydrat oder als Oxydul gefällt, and man erhält statt eines hell grünlichblauen eiuen dunkelblauen Niederschlag.

Der erhaliene Niederschlag wurde anf einem Filtrura gesammelt, mit destillirtem Wasser wohl ausgewaschen und im Wasserbade bei $100^{\circ}$ getrocknet, danu fein gepulıcrt. Er glich in seinem Auszehen volikommen dem durch scliwefelsaures Kupfer und füssiges Eiweifs erhaltenen Kupfersulze.

Un zu bestimmen, in welchem Verhältuisse die mit Kapferexyd verbundene organische Substauz Kohlenstoff. und Stiokatoff enthalte, wurde er mit Kupferoxyd verbrannt. In 12 Rölıren Gasgemenge war das durchschnittiche Verhältnifs des Stickstoff zur Kohlensïure wie 1 : 6,9. Eine zu Aufung der Verbrennung genommene Probe des Gasgenenges zeigte, dafs sich kein Stickstoffoxyd bildete; wohl aber liefs sich in einer an Ende deiselbell genommenen Probe, als sie mit atınosphärischer Luft gcmengt wurde, eine geringe Spur von salpetriger Säure wahrnehmen. Die letzten Röhren gaben also wegen einer geringeu Beimengung von Stickstoftoxyd den Stickstoffgehalt etwas zu hoch an. In den ersten 5 Röhren war das dnrchschnittliche Verhältnifs des Stickstoffs zur Kahlensäure wie $1: 7,30$, was geneu mit der oben angeführten Analyse des Kupferalbuminat übereiustimmt.

Wir dürfen aber daraus schliefsen, dafs das Eiweifs durch die künstliche Chymifikation in seiner Elementarzusammensetzung nicht wesentlich verändert wird, wiewohl es die Fähigkeit verliert, durch Hitze zu geriunen und durch Weingeist coagulirt zu werden.

Fasersiof. Frischer; noch nicht getrockneter Faserstoff löst sich leicht in der Verdauunganïssigkeit. Diefs ist auch in der Kälte bei gewöhulicher Zimmertemperatur nach 6--8 Stunden immer geschehen.

bic Auflösung ist trüb und lïfst sich nur schwer filtriren. 
49 Fogel, über einige Gegenstände der thierischen Chemie.

Kaliumeisencyønür bewirkt in de: sauren Flïssigkeit einen reichlichen weifsen, Kaliumcisencyanid einen hellgrünen Niederschlag, Querksilberchlorid einen weifsen.

Durch Weingeist entsteht eine kaum merkliche Trübung, durch Salpetersäure ein flockiger weifser Niederschlag.

Durch Neutralisation der Flissigkeit mit kohlensuren oder kaustischen Alkalien werden zarte weifse Flocken gefält, die sich aber beim Imschïuteln gröfstentheils wieder auflösen.

Gerbestoff bringt in dieser neutraitisirten Flüssigkeit elnen sehr reichlichen Niederschlag herror, auch in der sauren.

Durch schwefelsaures kupforoxyd eutsteht in der neutrnlen Flüssigkeit ein grïnlichblauer Niederschlag. Dieser wurde uäher untersucht und ganz so wie der aus dem chymificirten Eiweifs erhaltene behandelt. ifan hat bei seiner Darstellung dieselben Inrsichtsmafsregeln zu beobachten, welche bei jenem angegeben wurlen.

Ich verbranute ihn mit Kupteroxyd; beim Verbrennen liefs sinh keine $S_{\text {pur ron }}$ Stichstolloxyd wahrnehmen, ebenso wenig ein Geruch nach schwefliger siurure; - er enthält also keine Schwefelsäure. Da ich nur weuig substarz rerbrennen konnte, erhiclt ich auch nur wenige Rühren tiasgemenge; dieses enthieit aber Stickstoff und Kohterisäure in Verhältnifs von $1: 6,70$. Diel's ist aber gana genau dasscibe Verhältnils, wie ich es bein unveränderten Faserstofl fand.

Es geht also daraus hervor, difs auch der Faserstoff durch die künstiich Chymification keine Veränderung im Mengenrerhältnifs seiner klemente erleidet, wiewohl er die fähigkeit verliert, aus seiner wässerigen Aufösung con selbst zu gerinneı. 